\title{
Investigation of Factor Structure and Validation of the Parent - Infant Interaction Questionnair
}

\author{
Somayeh Habibpour ${ }^{1, *}$, Gholam Ali Afroz ${ }^{2}$, Mohsen Shokohi Yekta ${ }^{2}$, Mohammad Ali Besharat ${ }^{2}$, \\ Valio Alah Farzad ${ }^{3}$, Maryam Nakhshab ${ }^{4}$
}

${ }^{1}$ School of Psychology, Faculty of Psychology, Islamic Azad University of Neka Branch, Neka, Iran

${ }^{2}$ Department of Psychology, Faculty of Psychology, Tehran University, Tehran, Iran

${ }^{3}$ Department of Psychology, Faculty of Psychology, Kharazmi University, Tehran, Iran

${ }^{4}$ Department of Medical, Faculty of Medical, Mazandaran University, Sari, Iran

Email address:

Habibpour@iauneka.ac.ir (S. Habibpoor), afroz@ut.ac.ir (G. A. Afroz), msyekta@yahoo.com (M. S. Yekta), besharat2000@gmail.com (M. A. Besharat),vfarzad@yahoo.com (V. A. Farzad)

${ }^{*}$ Corresponding author

\section{To cite this article:}

Somayeh Habibpour, Gholam Ali Afroz, Mohsen Shokohi Yekta, Mohammad Ali Besharat, Valio Alah Farzad, Maryam Nakhshab. Investigation of Factor Structure and Validation of the Parent - Infant Interaction Questionnair. Biomedical Statistics and Informatics. Vol. 6, No. 3, 2021, pp. 65-71. doi: 10.11648/j.bsi.20210603.15

Received: May 4, 2021; Accepted: August 5, 2021; Published: August 23, 2021

\begin{abstract}
Background: the birth of a premature baby puts a lot of stress on the family. This stress is due to the lack of physical, emotional and psychological readiness of the parent to enter this premature baby and the family faces many needs, therefore, attention to the specific issues and needs of these families is considered. Objective: The purpose of the present study was to construct and investigate the factor structure and validation of the parent - infant interaction questionnaire in Behshahr. Method: After translation, design and approval of items by experts in the field of psychology and psychometrics, 222 mothers with infants over 2 months were selected by convenience sampling method and based on the criteria of Klein, moreover, this questionnaire was administered to them. Exploratory and confirmatory factor analysis were used to evaluate the construct validity. Result: According to the research findings, 3 factors and 19 items were identified. In addition, the confirmatory factor analysis confirmed the three - factor model of this questionnaire. To measure the reliability of the test, the KR-20 method was used, whose coefficients ranged from 0.610 to 0.803 . Conclusion: The results indicate that the questionnaire has desirable psychometric properties and is a good tool for measuring mother - infant interaction.
\end{abstract}

Keywords: Parent, Infant Interaction Questionnaire, Factor Structure, Reliability, Validity

\section{Introduction}

The family has a fundamental role in the mental and physical health of the infant; furthermore, the optimal and healthy development of children in all aspects of psychosocial owes to this basic educational base. Moreover, one of the most significant factors affecting the mental and social development of children is optimal parent - infant interaction [1].

Parenting is a complex and challenging process [2] which itself is composed of many numerous factors such as parental attitude, acceptance amount, method of managing child behavior, parental knowledge, skills, parental confidence, good thinking, sense of social competence, self - control and creation of environment with least conflict [3]. This interaction is the first representative of the awareness of the child form communication world, which is very important and vital for receiving love and security from parents [4]. Moreover, the quality of this interaction in the early years of childhood also guarantees the child's cognitive, social and emotional development [5].

The results of research on the importance of the early years of childhood, have led to increasing attention of researchers to early experiences and especially the child's interaction with caregiver. Parent - infant supportive interactions can ensure the child's developmental path, especially the 
acquisition of social skills, mental health and social competence [6].

Bilateral parent - infant communicative interactions play an important role in the development or reduction of behavioral problems; in addition, any intervention in this area is very necessary and vital, and also brings interactive effects for parents and children [7].

Numerous studies have shown that undesirable and dysfunctional interactions during the developmental period lead to communication problems and ultimately vulnerability of children to disorders including anxiety, behavioral problems and psychological damage [8]. In addition, failure to form a secure attachment in the early years of life can have negative and detrimental effects on physical and cognitive emotional development in childhood and adulthood attachments [9].

It has also been confirmed that children diagnosed with behavioral problems such as delinquency, aggression, and coping disobedience often have attachment problems and a history of abuse, neglect, and misbehavior [10].

Stable and efficient parent - child interaction is not only suitable for the normal psychological and social development of the child, but also in the following years, is a source for child's vital development. Since the efficient and proper functioning of attachment provides child with an important resource for coping with stress and using problem - solving methods and flexibility and resilience [11]. Children who have had warm and rich parenting will become capable, creative and adaptable adults in the future [5]. In other words, the family environment is the best predictor of maladaptive and delinquent behaviors of adolescents [12].

In this regard, reviewing various empirical evidence indicated that the study of factors explaining the improvement of parent - child interaction is one of the most important concepts of researchers in the field of family psychology; moreover, its various aspects have always been emphasized $[13,14]$.

Improvement of the quality of parent - child interaction is associated with reducing child behavioral problems and increasing societal behaviors, improving parenting skills such as firm discipline and reducing parental stress [15]. The quality of parent - child interaction is effective in regulating children's social and executive functions or changing the parent - child interactive styles which leads to preventing parent - child interaction problems, strengthening parenting practices and improving parental self - confidence [16].

It is also important to note that children's psychological, emotional, and behavioral problems will not be cured unless accurate screening methods and appropriate assessment are designed to identify the problematic parent - child interactions. In other words, the first step of effective interventions is accurate measurement and design of valid and reliable tools in this field so that accurate diagnosis and measurement is a significant part of treatment [17].

During to the review of research literature in this field, we found that there is no suitable tool to measure the parent - child interaction in infancy (under two years); moreover, there are many research gaps in this field, so in the present study, we seek to design and standardize a suitable tool for measuring the interaction of the infant - parent (under two years old) that firstly covers the existing research shortcomings in this field and secondly takes an important step in the implementation of clinical interventions in this period. It should be noted that other tools in this field, such as the Pianta scale 1994 [5]. the parent - child interaction questionnaire by Fine, Moreland and Schwbel 1983 [4] and the parent - child interaction questionnaire by Gerard 1994 [6] evaluate the parent - child interaction at the age over 3-4 years, which is not appropriate for infancy (under 2 years).

\section{Research Method, Population and Statistical Sample of the Research}

The present study was a descriptive-applied and psychometric research whose results will be used in practice by experts and researchers. Considering that in the current study, the purpose of constructing and validating the questionnaire was parent - infant interaction; moreover, a kind of product was presented. Therefore, it is in the category of research and development.

To achieve the objectives of the study (psychometric properties of the scale), content validity (expert opinion), construct validity (exploratory and confirmatory factor analysis) and Cronbach's alpha coefficient were used to evaluate the reliability. The statistical population of the current study includes all mothers with infants 2 months and older who have referred to health centers in Mazandaran province to receive health care and vaccination services that were selected among 222 people available by sampling method according to Klein criteria 2001 [18].

\section{Instrument}

According to the theoretical foundations of research and research literature in the field of parent - child interaction, child developmental psychology and the help of specialized professors of psychology and psychometrics, first 50 appropriate items were designed for this period.

Next, a set of items in the form of a 50-item scale was provided to five experts in the field of child psychology. The desired and useful items were selected and other unnecessary options that were related to older children were identified and [10] items were removed. Finally, 40 items remained; in addition, a form was prepared to determine the face validity with the opinion of psychometricians. Furthermore, items were examined psychologically, transparently, comprehensibly and in accordance with the cultural conditions of the Iranian society based on the opinion of 5 psychrometers. If $80 \%$ of the participants had a score (perfectly proportional) for each item, that item was accepted and otherwise the items were removed [19].

It should be noted that none of the items were deleted. Then the final scale was executed in the sample; moreover, the data were analyzed in SPSS / Liserl software. As 
mentioned, the sample consisted of 222 people who were selected by convenience sampling and divided into two equal groups of 111 people based on even and odd numbers. The purpose was the initial analysis and exploratory factor analysis for the first sample and the confirmatory investigation of the extractive model and the confirmatory factor analysis in the second sample.

To evaluate the content validity of the scale, the two coefficients of content validity ratio (CVR) and content validity index (CVI) have been used quantitatively. To determine the content validity ratio, a total of ten experts were asked to examine each item based on a 3 - likert scale (fully relevant, somewhat relevant, not relevant at all) and calculate

its value based on the following formula. The content validity calculated for each items based on the Lawshe table for ten people should be more than 0.42. [20]. Table 1 lists the CVR values and the post-calculated result for each dimension. At this stage, 10 items were removed because they did not get the required points; moreover, 30 items remained.

$$
C V R=\frac{n E-\frac{N}{2}}{\frac{N}{2}}
$$

Table 1. Content validity of the questionnaire.

\begin{tabular}{|c|c|c|c|c|c|c|c|c|}
\hline Item & CVR & Result & Item & CVR & Result & Item & CVR & Result \\
\hline 1 & $81 \%$ & Accepted & 8 & $85 \%$ & Accepted & 15 & $85 \%$ & Accepted \\
\hline 2 & $94 \%$ & Accepted & 9 & $91 \%$ & Accepted & 16 & 1 & Accepted \\
\hline 3 & 1 & Accepted & 10 & 1 & Accepted & 17 & $95 \%$ & Accepted \\
\hline 4 & $75 \%$ & Accepted & 11 & $95 \%$ & Accepted & 18 & 1 & Accepted \\
\hline 5 & $78 \%$ & Accepted & 12 & 1 & Accepted & 19 & $85 \%$ & Accepted \\
\hline 6 & 1 & Accepted & 13 & $98 \%$ & Accepted & & & \\
\hline 7 & $94 \%$ & Accepted & 14 & 1 & Accepted & & & \\
\hline
\end{tabular}

Then, to determine the content validity index, three criteria of simplicity, specificity (relevance) and clarity were evaluated as a 3-Likert scale (appropriate, somewhat appropriate, not appropriate at all) for each item by 10 experts. These scores were calculated by addition of the agreed scores for each item that ranked 3rd and 4th, on the total number of specialists. In addition, the acceptance limit was based on a score above $79 \%$ [20].

\section{Findings}

Table 2 shows the demographic information of the sample group in terms of age, gender, pregnancy and place of living.

Table 2. Demographic characteristics of the subjects.

\begin{tabular}{llll}
\hline \multirow{2}{*}{ Variables } & Demographic factors & Sample & \\
\cline { 3 - 4 } Age & $20-30$ years & 164 & 73.9 \\
& $31-40$ years & 58 & 26.1 \\
Gender & Male & 105 & 47.3 \\
& Female & 117 & 52.7 \\
Pregnancy & First & 89 & 40.1 \\
\multirow{2}{*}{ Place of living } & Second & 133 & 59.9 \\
Total -222100 & City & 161 & 72.5 \\
\hline
\end{tabular}

Bartlett Exam was used to evaluate the adequacy of correlation matrix for exploratory factor analysis. Based on the exploratory factor analysis, (KMO) is 0.669 and the significance level of the Bartlett's test of sphericity is 0.000 , which indicates that the sample is suitable for factor analysis Table [3].

Then, to determine the construct validity of this questionnaire, factor analysis method was used, which is one of the statistical methods of multivariate analysis. First To perform factor analysis, the fit and adequacy of the correlation matrix were examined and the sampling adequacy value was 0.699 .

Bartlet's test of sphericity was significant at the level of more than one per thousand $(\mathrm{P}<0.000)$, which indicates that the sphericity hypothesis has been rejected; moreover, the data correlation is not zero. Therefore, the correlation matrix is sufficient for exploratory analysis.

Table 3. Adequacy of sampling.

\begin{tabular}{ll}
\hline Adequacy of sampling (KMO) & 0.669 \\
Bartlett's test of sphericity (BTS) & 2013.618 \\
Sig. & 0.000 \\
\hline
\end{tabular}

Data analysis in the present study was performed in two general stages. After evaluating and ensuring the accuracy of the data and making the necessary corrections, the analysis process entered in to the main stage of data analysis. As shown in Table 4, the values of the three factors are greater than 1 or the factors have more than 3 items. Based on the obtained values, the existence of three factors in parallel analysis in sub - scale exploration was confirmed; in addition, the equation of 19 items was analyzed and the above factors were confirmed and finally 3 confirmed factors explained 99.65 of the total variance. In addition, in the present analysis, due to the correlation of the items, they have been rotated by the Promax method. The results of Promax rotation with the logical model and theoretical foundations show the greatest fit. At this stage, 11 items that had a low correlation with the whole test were removed from the analysis and 19 items remained. Items with a low correlation coefficient (less than 0.3 ) are removed at this stage [21]. Thus, only items that provide valid information were remain Table [4]. Cronbach's alpha increased to 0.86 after removing the defective items, which according to Nunnally's criteria, values above 0.7 are acceptable. Thus, the conditions and requirements for 
entering the next step of tool cleaning (exploratory factor analysis) were provided.

Table 4. Mean and percentile 95 eigenvalues of random data and real data.

\begin{tabular}{llllll}
\hline Factor & $\begin{array}{l}\text { Real special } \\
\text { value }\end{array}$ & $\begin{array}{l}\text { Mean of special eigenvalues } \\
\text { of random data }\end{array}$ & $\begin{array}{l}\text { Percentile 95 eigenvalues of } \\
\text { random data and real data }\end{array}$ & $\begin{array}{l}\text { Percentage of variance } \\
\text { explained by each factor }\end{array}$ & $\begin{array}{l}\text { Compression } \\
\text { percentage }\end{array}$ \\
\hline Emotional behavior & 5.300 & 3.585 & 2.844 & 42.38 & 42.38 \\
Caring behavior & 4.458 & 3.152 & 2.585 & 30.52 & 72.90 \\
Proximity behavior & 3.896 & 2.766 & 2.154 & 24.79 & 97.65 \\
\hline
\end{tabular}

\section{Rotation of Principal Components with Varimax Rotation}

Based on Table 5, the factor load of all items on the factors is higher than 0.04, which indicates that the items are significantly related to their underlying factor, so it can be said that all items are related to the specified factors. For example, items 1 - 9 are related to the first factor, items 15 10 are related to the second factor and items $16-19$ are related to the third factor, which also indicates the validity of the appropriate prediction.

Table 5. Final extracted factors after removing defective items.

\begin{tabular}{llll}
\hline Question & The first factor (emotional behavior) & The second factor (caring behavior) & The third factor (proximity behavior) \\
\hline 1 & 0.758 & & \\
2 & 0.829 & & \\
3 & 0.574 & & \\
4 & 0.782 & & \\
5 & 0.816 & & \\
6 & 0.528 & & \\
7 & 0.510 & 0.896 & \\
8 & 0.615 & 0.622 & 0.832 \\
9 & 0.642 & 0.705 & 0.920 \\
10 & & 0.886 & 0.882 \\
11 & & 0.765 & 0.858 \\
12 & & 0.550 & \\
13 & & & \\
14 & & & \\
15 & & & \\
16 & & & \\
17 & & & \\
18 & & & \\
19 & & & \\
\hline
\end{tabular}

\section{Factor Naming}

Complementary activity after extraction of underlying factors is factor naming. The importance of this action is related to the unification and determination of common concepts among the questions that make up the factors. Since items [1] to [9] include concepts related to the emotional aspects of the parent - infant interaction are called emotional behaviors. As items [10] to [15] related to infant care concepts are called caring behaviors.

Items [16] to [19] are called proximity behaviors because they include concepts related to closeness and proximity to the infant.

In order to answer the question of whether the factor structure obtained from the first sample in the second sample is confirmed, the analysis has entered in to the second stage. The second stage is to review, confirm, modify or reject the factor structure extracted from the exploratory factor analysis stage to the confirmatory factor analysis stage. If the structure obtained from the first sample is also confirmed in the second sample, a certificate for its validity is also provided. The data entered in this step are the second ran dom sample; however, the defective items identified in the piervius step before this perep have been removed in this step.

Table 6. Analysis results of measurement models and lateral evaluation indicators.

\begin{tabular}{|c|c|c|c|c|}
\hline Subcomponent & Standard factor load (P. S) & Mean factor loads (T) & Determination coefficient $(\mathrm{R})$ & Result \\
\hline Emotional behavior & 5.55 & 40.4 & 5.01 & Significant \\
\hline Caring behavior & 5.45 & 53.18 & 5.6 & Significant \\
\hline Proximity behavior & 5.63 & 43.2 & 5.01 & Significant \\
\hline
\end{tabular}

As can be seen in Table 6, the standard factor coefficient of the components of emotional behaviors, proximity behaviors and caring behaviors in explaining the parent - infant interaction is equal to (5.55), (5.45), (5.63) respectively.
Therefore, according to their factor loads, which are all greater than 1.96; therefore, it can be concluded that all components of the parent - infant interaction questionnaire are effective in the final explanation of parent - infant interaction. 


\section{Confirmatory Factor Analysis}

Table 6 illustrates the results obtained from the measurement models and measurement indices of the relationships of each factor with its markers. The standard factor load, $T$ value and percentage of variance explained for each indicator of each factor are specified in Table 6. Evaluation index, the degree of relationship of each indicator to its underlying factors, a value of $\mathrm{T}$ above 2 indicates the significance of the relationship between each indicator and the related factor. As shown in the table, for all the relationships of the markers with their factors, the amount of $\mathrm{T}$ is above 2. Therefore, it is concluded that all indicators are significantly related to their underlying factor and it can be said that the measurement model is desirable during the evaluation of partial indicators.

After confirmatory factor analysis, we examined the adequacy of the data processing model. It is noteworthy that among the various indicators of the suitability of the model, the root mean square of the estimation error and the goodness of the fit were among the best and most adequate models.

The following table illustrates the fit were among indices of the analysis model in the causal model of the research.

According to Table 7, which shows the goodness-of-fit indices and the criterion as a (desirability) in the three tests of goodness-of-fit index, standardized fit index, adaptive fit index is greater than and equal to 0.9 . These values are above the desired level, which indicates the confirmation of these three indicators. In addition, the root of the error of the mean squared approximation is less than 0.8 , which is still reported to be desirable. Moreover, the ratio of chi - square to degrees of freedom is less than 3. In general, the level of value obtained in line with the desired values in the indicators is a good fit of the Figure 1.

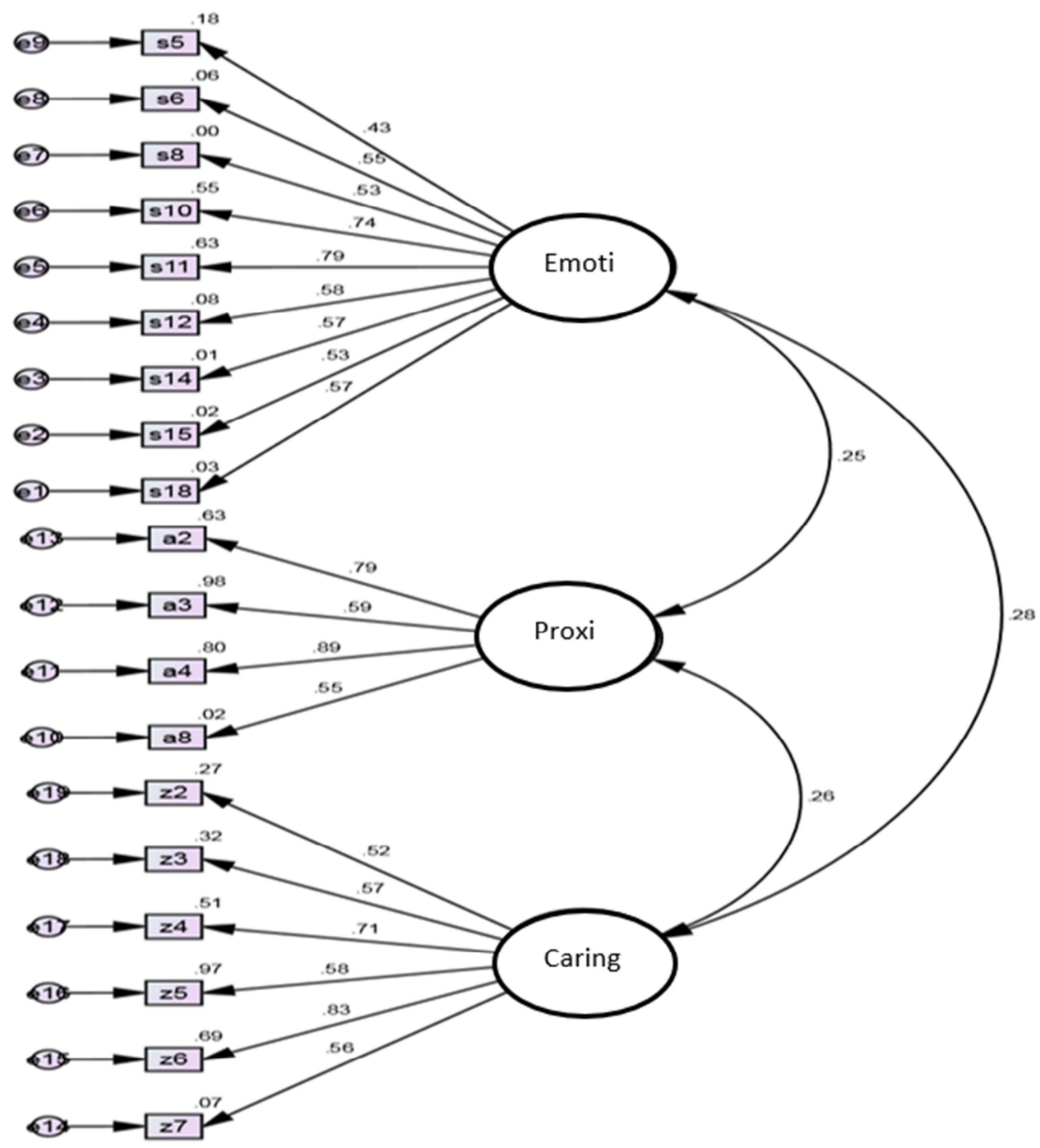

Figure 1. Construct validity of the tested model with standardized predicting statistics.

In order to obtain more reliability of Promax rotation test, factor analysis in construct validity was also used. In general, the study of construct validity of all three - factor items indicates that the factor loads of none of the three - factor items are lower than 0.30 , which is related to the fact that all values are higher than 0.3 , The construct validity is confirmed. In addition, the fit indices in the table below confirmed the above results. 
Table 7. Fit indicators obtained from analysis of data and variables.

\begin{tabular}{lll}
\hline Goodness of fit index & Optimal values & Obtained values \\
\hline Goodness of fit index (GFI) & $90 \geq 0$ & 0.996 \\
Normed fit index (NFI) & $90 \geq 0$ & 0.989 \\
Comparative Fit Index (CFI) & $90 \geq 0$ & 0.984 \\
Root - Mean - Squared Error of approximation (RMSEA) & $0.8 \leq 0$ & 0.038 \\
Ratio of chi - square to degrees of freedom ( $/ \mathrm{df})$ & $\geq 3$ & 2.245 \\
(P) value & $0.05 \geq 0$ & 0.000 \\
\hline
\end{tabular}

According to Table 8, the optimal criterion of reliability by the internal consistency method through Richardson Kuder is equal to 0.5 , which in the present study, the obtained factors and the total amount of reliability have indicated their reliability.

Table 8. Results of reliability analysis of the studied structures along with markers.

\begin{tabular}{ll}
\hline Factor & Kuder - Richardson $\geq \mathbf{0 . 5}$ \\
\hline Emotional behavior & 0.610 \\
Caring behavior & 0.783 \\
Proximity behavior & 0.803 \\
Total & 0.709 \\
\hline
\end{tabular}

Another method of estimation is the reliability of intragroup correlation with test components [18] which was carried out in the current study through Pearson correlation. The results in Table 9 show a significant correlation between emotional behavior, caring behavior, proximity behavior and total score, which is significant at the level (0.01).

Table 9. Pearson correlation coefficient matrix between subscales or overall score.

\begin{tabular}{lllll}
\hline Variable & 1 & 2 & 3 & 4 \\
\hline Emotional behavior & 1 & & & \\
Caring behavior & $.24 * *$ & 1 & & \\
Proximity behavior & $.31 * *$ & $.47 * *$ & 1 & \\
Total & $.72 * *$ & $.73 * *$ & $.79 * *$ & 1 \\
\hline
\end{tabular}

\section{Discussion and Conclusion}

The purpose of the present study was to investigate the factor structure and validation of the parent - infant interaction questionnaire in Iranian context. The results indicate that the parent - infant interaction questionnaire has good reliability and is also reliable. More ever This scale has good validity evidence so that it can be used as a suitable questionnaire in clinical interventions in the neonatal period.

The results of construct validity analysis through exploratory factor analysis showed that this questionnaire consists of three components "emotional behaviors, proximity behaviors and caring behaviors"; moreover, the distribution of questionnaire items is consistent with the main test. These three factors together explained $99.65 \%$ of the total variance in the questionnaire. The available factor load matrix indicates that all extracted factor loads are higher than 0.4; in addition, the distribution of items in the factors corresponds to the main test. In the current study, the results of confirmatory factor analysis confirmed the three - factor model. All significant coefficients were obtained; furthermore, all fitness indices were in the desired range.

The internal consistency of this questionnaire was reported by using Cronbach's alpha of 0.709 . In addition, in order to validate the structure, the scale was conducted by the internal consistency method by KR - 20 method; moreover, the inter group correlation was carried out by Pearson correlation coefficient method.

Considering that in Iran, a suitable tool for measuring parent - infant interaction is not designed, the designed items of the present tool in the field of parent - infant interaction are valid and reliable so that in future studies, a step can be taken by using this tool in order to determine the accurate and comprehensive parent - infant inter action, which provides reliable information for planning and elimination of deficiencies and shortcomings by researchers and therapists. Moreover, this tool can improve the quality of treatment and services provided to mothers in the best way.

Finally, repeating the factor structure of this scale in other regions of Iran or larger samples in order to evaluate the diagnostic validity of this scale in the measurement, and investiging the effectiveness of this scale in evaluating the results of treatments and psychological interventions based on improving mother - infant interaction are suggested in future studies.

\section{Acknowledgements}

We would like to express our gratitude to all the loved ones who have helped us in this research, especially the officials of the health centers of Mazandaran province.

\section{References}

[1] Allah Panah, S. (2019). Family and Its Importance, Journal of Preschool Education Development, 10 (3).

[2] Aman Elahi, A., Shadfar, A., Aslani, Kh. (2021). The Effectiveness of the Parent - Child Relationship on Mothers' Parenting Stress and Parental Acceptance of Primary School Children, 41 (23).

[3] Panahipour, H. Hosseinian, S., Ghasemzadeh, S. (2018). The Effectiveness of Timely Support Program on Intimate Promotion and Parent - Child Interaction,. Bi - Quarterly Journal of Contemporary Psychology, 13 (1).

[4] Mirzaei Kontaie, F., Shakerinia, I., Asghari, F. (2015). Relationship between parent - child interaction and the level of aggressive behaviors, Quarterly Journal of Child Mental Health, 2 (4). 
[5] Adili, Sh., Mirzaei Ranjbar, R., Abedi, A. (2009). The Effectiveness of Parent - Child Relationship Play Therapy on the Quality of Parent - Child Interactions and the Quality of Life Related to the Health of Children with Behavioral and Emotional Problems. Exceptional Children Quarterly, 19 (1).

[6] Shokouhi Yekta, M., Parand, A., Dargahi, M. (2016). Family centered preventive interventions: The effect of parent - child relationships and parenting methods. Child Mental Health Quarterly, 2 (3).

[7] Akbarizadeh, A., Hassanzadeh, S., Kamkari, S., Lavasani, Gh. A. (2017). The effectiveness of face-to-face combined education in parent - child interaction based on Eyberg approach on mother - child relationship in children with behavioral disorders. Quarterly Journal of Applied Psychology Research, 11 (10).

[8] Patterson GR. The early development of coercive family process. in J. Reid G. R. Patterson \& J. Snyder (Eds.). Antisocial behavior in children and adolescents: $A$ developmental analysis and model for intervention. Washington DC: American Psychological Association; 2010, pp: $22-44$.

[9] Ali Qanavati, S., Sharifi, T., Ghanzanfari, A., Abedi, M. R., Taghavi, A. (2009), The effectiveness of the parent - child interaction training package on the quality of attachment of children with dysfunctional mother - child interaction, 10 (2).

[10] Choi EJ. Taylor MJ, Hong S, Kim C, Yi S. The neural correlates of attachment security in typically developing children. Brain and cognition; 2018, pp: 47-56.

[11] Wymbs BT. Pelham WE. Child Effects on communication between Parents of Youth with and without ADHD. J Abnorm Psychol. 2018; 119 (2): 366-375. 14.

[12] Brewer JD, Sparkes AC. Young people living with parental bereavement: Insight from an ethnographic: 2018. pp. 112-115.
[13] Bayazit, I. An Investigation of Problem Solving Approaches, Strategies, and Models Used by the 7th and 8th Grade Students when Sloving Real - World Problems. Educational sciences: Theory \& Practice: 2013. pp. 1920-1927.

[14] Shokoohi Yekta, M., Shahabian, A., Parand, A. (2012). Family Psychology, Tehran: Teymourzadeh Publications.

[15] Damodaran KD. Parent child interaction therapy: Child Well being through an affective parenting intervention, the journal of positive psychology. 2013: 35 (2): 45-53.

[16] Sheperis C, Sheperis D. Monceaux A. Davis JR. Lopez B. Parent - child interaction therapy for children with special needs: The professional counselor: 2015. pp: 32-45.

[17] Gump BG, Dykas MJ, Mackenzie JA, Dumas AK, Hruska B, Ewart CK, Parson PJ. Palmer CD, Bendinskas K. Abnormality in children: 2017. pp: 23-31.

[18] Klin RB. Principles and Practice of Structural Equation Modeling 3rd ed. New york: The Guilford press; 2011, pp: 23-31.

[19] Jensen MP Questionnaire validation: a brief guide for readers of the research literature. Klin J Pain: 2003, pp: 345-352.

[20] Nadighara A, Abedi G, Abedi E. Rostami E. Designing and validating to Measure the security in Hospitalized patients. Journal Mazandaran univ med. 2016; 26 (139): 178 189. persian )

[21] Nunnally JC, Bernstein IH. Psychometric theory. 3rd. New york: Mc Graw Hill; 2012, pp: 17-23.

[22] Saleh Sadeghpour, B., Ebrahim Damavandi, M., Vaedi, A. (2017). Reconstruction, validation, reliability, standardization of mathematical self-concept scale in high school students. Educational Measurement Quarterly, 8 (30). 